\title{
Spin splitting in a polarized quasi-two-dimensional exciton gas
}

\author{
L. Viña, L. Muñoz, and E. Pérez \\ Departamento de Física de Materiales, C-IV, Universidad Autónoma de Madrid, Cantoblanco, E-28049, Madrid, Spain \\ J. Fernández-Rossier and C. Tejedor \\ Departamento de Física Teórica de la Materia Condensada, Universidad Autónoma de Madrid, Cantoblanco, E-28049, Madrid, Spain \\ K. Ploog \\ Paul Drude Institut fuer Festkoerperelektronik, Hausvogteiplatz 7, Berlin, D-10117, Germany
}

(Received 8 July 1996)

\begin{abstract}
We have observed a large spin splitting between "spin" +1 and -1 heavy-hole excitons, having unbalanced populations, in undoped GaAs/AlAs quantum wells in the absence of any external magnetic field. Time-resolved photoluminescence spectroscopy, under excitation with circularly polarized light, reveals that, for high excitonic density and short times after the pulsed excitation, the emission from majority excitons lies above that of minority ones. The amount of the splitting, which can be as large as $50 \%$ of the binding energy, increases with excitonic density and presents a time evolution closely connected with the degree of polarization of the luminescence. Our results are interpreted on the light of a recently developed model, which shows that, while intraexcitonic exchange interaction is responsible for the spin relaxation processes, exciton-exciton interaction produces a breaking of the spin degeneracy in two-dimensional semiconductors. [S0163-1829(96)53036-5]
\end{abstract}

The spin dynamics of low-dimensional semiconductor heterostructures has been studied intensively both experimentally $^{1-6}$ and theoretically ${ }^{7-9}$ in the past decade. One of the most intriguing findings, first observed by Damen et $a{ }^{1}{ }^{1}$ in time-resolved photoluminescence (TRPL) spectra, is the appearance of an energy splitting between excitons with spin +1 and $-1,{ }^{10}$ in the absence of any magnetic field, whenever circularly polarized light is used to excite the system. The breaking of the spin degeneracy at high excitonic densities was confirmed by pump-and-probe experiments, ${ }^{2}$ and later on by TRPL. ${ }^{3,4,11}$ Closely related to this behavior of spin-polarized excitons is the existence of a blueshift of excitonic transitions, observed in pump-andprobe experiments in GaAs quantum wells (QW's) under high excitation. ${ }^{12,13}$ This shift has been attributed to the repulsive interaction among excitons due to the Pauli exclusion principle acting on the Fermi particles forming the excitons. ${ }^{14,15}$ In a recent publication, ${ }^{9}$ we have shown that, in the case of spin-dependent populations, interexcitonic interaction produces a breaking of the spin degeneracy in twodimensional excitons. This mechanism is complementary to the intraexciton exchange, which induces the spin relaxation. ${ }^{8}$

We present in this work new experimental results on spin splitting of heavy-hole (hh) excitons in intrinsic GaAs quantum wells and compare the energies of the interactingexcitons system with theoretical calculations based on the model described in Ref. 9. We have used a sample consisting of 50 periods of $77-\AA$-wide GaAs wells and $72-\AA$-wide AlAs barriers, which presents a small Stokes shift between the emission and the absorption $(2.5 \mathrm{meV}$ at $2 \mathrm{~K}$ and very low exciting power). The Stokes shift allows us to perform quasiresonant excitation experiments, i.e., detect in the peak of the photoluminescence while exciting in the ground-state absorption peak. In spite of the presence of this shift, the sample exhibits dynamical properties comparable to those of very-high-quality samples. ${ }^{6}$

TRPL spectra have been measured with a standard upconversion setup, using a double monochromator to disperse the up-converted signal. The exciting light, obtained from a Styryl 8 dye laser synchronously pumped by the 532-nm line of a mode-locked Nd:YAG laser, was circularly polarized by means of a $\lambda / 4$ plate, and the photoluminescence (PL) was analyzed into its $\sigma^{+}$and $\sigma^{-}$components using a second $\lambda / 4$ plate before the nonlinear crystal, where the upconversion takes place. Spectra were taken at different times delays with the sample mounted in the cold finger of a temperature-variable cryostat. The time resolution of our system is 5 ps.

Figure 1 depicts time-resolved PL spectra at $8 \mathrm{~K}$ taken 10 ps after the excitation with $\sigma^{+}$pulses for two different densities, $4 \times 10^{10}$ and $1.5 \times 10^{11} \mathrm{~cm}^{-2}$, with the laser at 1.625 $\mathrm{eV}$. The solid points show the polarized $\left(\sigma^{+}\right.$, spin +1$)$ emission while the open circles correspond to the unpolarized $\left(\sigma^{-}\right.$, spin -1$)$ luminescence. With this quasiresonant excitation, an energy splitting of $2.5 \mathrm{meV}$ is clearly seen between the two peaks in Fig. 1(a). Increasing the excitation density, both a broadening of the lines, which increases from 12 to 18 $\mathrm{meV}$, and a strong enhancement of the splitting is observed. The splitting is mostly due to the redshift of the $\sigma^{-}$polarized emission and exhibits marked time and excitation-energy dependences.

The time dependence of the polarized (solid points) and unpolarized (open points) photoluminescence is shown in Fig. 2 for two different excitation energies and an initial carrier density of $5 \times 10^{10} \mathrm{~cm}^{-2}$. At short times, the splitting amounts to $4 \mathrm{meV}$ exciting at $1.631 \mathrm{eV}$, below the light-hole (lh) exciton (diamonds). However, if the excitation is moved 


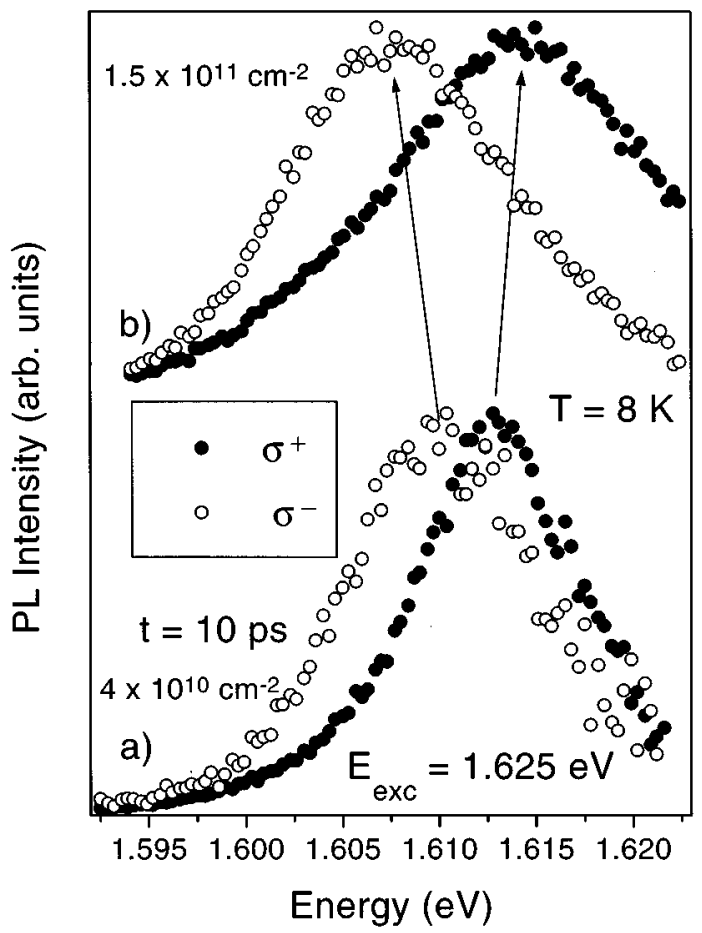

FIG. 1. Low-temperature, $8 \mathrm{~K}$, time-resolved PL spectra of a 77$\AA$-wide GaAs QW taken 10 ps after the excitation with $\sigma^{+}$polarized light at $1.625 \mathrm{eV}$. The solid (open) points depict the $\sigma^{+}$ $\left(\sigma^{-}\right)$emission. Initial carrier density: (a) $4 \times 10^{10} \mathrm{~cm}^{-2}$, (b) $1.5 \times 10^{11} \mathrm{~cm}^{-2}$. The arrows indicate the blue(red)shift of the $\sigma^{+}$ $\left(\sigma^{-}\right)$luminescence.

at energies above the $\mathrm{lh}$ it becomes only $1.2 \mathrm{meV}(1.681 \mathrm{eV}$, circles). We have found that the splitting is strongly correlated with the degree of polarization $(P)$ of the luminescence. Time-resolved measurements on the same sample have shown that $P$ at $t=10$ ps amounts to $80 \%$ and $20 \%$ at $1.631 \mathrm{eV}$ and $1.681 \mathrm{eV}$, respectively. ${ }^{6}$ The behavior of the peak positions of the PL with time, seen in the figure, is common for all excitation energies: the polarized (unpolarized) emission shifts towards lower (higher) energies with increasing time until both emission bands merge at $\sim 150 \mathrm{ps}$.

Figure 3 shows the dependence of the energy positions of the luminescence on the initial carrier density. The numbers in the abscissa have been estimated from the absorption coefficient of the sample, the power density, the area in which the laser beam is focused, and the losses in the windows of the cryostat, and are subjected to substantial uncertainties. Under the conditions presented in the figure, 12 ps after the excitation at $1.631 \mathrm{eV}$, the $\sigma^{+}$emission remains practically constant, while the $\sigma^{-}$luminescence redshifts with increasing carrier density up to $\sim 7 \times 10^{10} \mathrm{~cm}^{-2}$. For higher carrier densities the energy of the unpolarized emission stays constant. The solid lines correspond to the calculation of the excitonic energies according to the method described in Ref. 9, which takes into account interexcitonic exchange interaction and screening. The changes in the energies of the interacting +1 and -1 excitons with respect to the energy of a single exciton are written analytically as

$$
E^{ \pm}=2\left(n^{ \pm}+f n^{\mp}\right)\left(I_{1}-I_{2}\right)-\frac{0.82 \pi e^{2} n a}{\epsilon},
$$

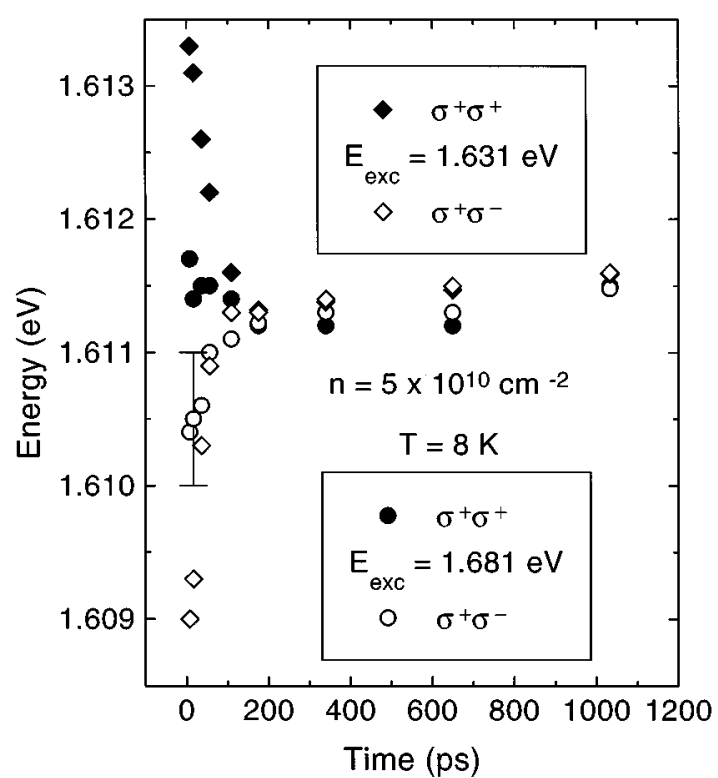

FIG. 2. Time evolution of the excitonic energies for $\sigma^{+}$(solid points) and $\sigma^{-}$(open points) emission at two excitation energies: $1.631 \mathrm{eV}(\diamond)$ and $1.681 \mathrm{eV}(\bigcirc)$, for an initial carrier density of $5 \times 10^{10} \mathrm{~cm}^{-2}$. The exciting light was $\sigma^{+}$polarized.

where $n$ is the total density of excitons, $n^{ \pm}$are those of \pm 1 excitons, $\epsilon$ the dielectric constant and $a$ the threedimensional Bohr radius. $I_{2}$ describes a "self-energy" correction that weakens electron-electron and hole-hole repulsion. $I_{1}$ is a "vertex" correction that, due to the Pauli exclusion, reduces the interexcitonic electron-hole attraction. The term involving $f$ is a small coupling between \pm 1 excitons, essentially due to valence-band mixing. Since a $+3 / 2$ $(-3 / 2)$ hole has a small coupling with a $-1 / 2(+1 / 2)$ hole, a hh $+1(-1)$ exciton has weight mainly on $-1 / 2(+1 / 2)$ electrons but also some weight on $+1 / 2(-1 / 2)$ electrons. The last term in Eq. (1) is a screening correction using the random-phase approximation.

Using the expressions for $I_{1}$ and $I_{2}$ given in the Appendix of Ref. 9, with $\epsilon=13$ for GaAs and neglecting the small $f$ term, Eq. (1) can be rewritten as

$$
\begin{aligned}
E^{ \pm}(\mathrm{eV})= & 2.214 \times 10^{-16} a(\AA) \\
& \times\left[1.515 n^{ \pm}\left(\mathrm{cm}^{-2}\right)-0.41 \pi n\left(\mathrm{~cm}^{-2}\right)\right]
\end{aligned}
$$

In the lines of Fig. 3, the energy of a single exciton, corresponding to the limit $n \rightarrow 0$, has been taken as the experimental energy of the +1 exciton at the lowest carrier density used in the experiments $\left(\sim 6.5 \times 10^{9} \mathrm{~cm}^{-2}\right)$. A three-dimensional excitonic Bohr radius of $150 \AA$ and an initial degree of polarization $P=80 \%$, corresponding to $n^{+}=0.9 n$ and $n^{-}=0.1 n$, have been used to compute the curves. The assumption that $P$ does not depend on $n$ is corroborated by previous TRPL experiments. ${ }^{6}$ The results are plotted up to $9.5 \times 10^{10} \mathrm{~cm}^{-2}$, where a saturation of the splitting of the photoluminescence is observed.

The theory obtains a very good agreement with the experiments for $\sigma^{+}$emission and agrees qualitatively with the 


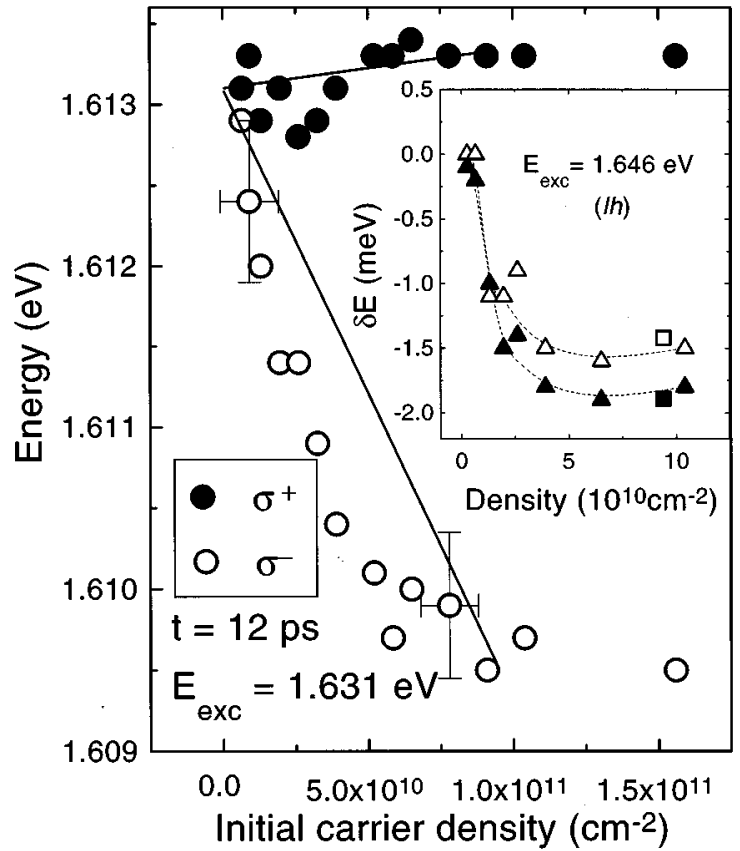

FIG. 3. Energies of the polarized ( $\sigma^{+}$, solid points) and unpolarized ( $\sigma^{-}$, open points) luminescence as a function of carrier density. The positions are taken $12 \mathrm{ps}$ after the excitation at 1.631 $\mathrm{eV}$. The lines represent the results of Eq. (2). The inset shows the dependence on carrier density of the split luminescence at $10 \mathrm{ps}$ after the excitation at the light-hole exciton energy. The squares show the predictions of the theoretical model and the dashed lines are a guide to the eye.

dependence of the $\sigma^{-}$luminescence. The flatness of the calculated position for the polarized $\left(\sigma^{+}\right)$PL arises from an almost perfect cancellation of the self-energy, the vertex correction, and the screening in Eq. (2) for $E^{+}$. On the other hand, the theory predicts a less pronounced redshift of the unpolarized $\left(\sigma^{-}\right)$PL with increasing carrier density than that observed experimentally, and does not reproduce the saturation of the shift. These discrepancies between the experiments and the calculations can originate from different sources: (i) the theory depends on excitonic density, while the experiments are plotted against carrier density, which is not necessarily the same as the excitonic one, especially at high densities when the number of created excitons saturates; (ii) the densities have considerable uncertainties in their estimation; (iii) the theory assumes free excitons with zero kinetic energy, while the excitons are actually weakly bound, ${ }^{6}$ and have some excess energy because they are not strictly created under resonant conditions; (iv) the theory considers a strictly two-dimensional system while the actual one has a width in the growth direction. This last approximation distorts the relative importance of exchange versus Hartree interaction.

The interdependence between the magnitude, and also the sign, of the splitting and $P$ is further demonstrated in the inset of Fig. 3, which depicts the shift of the PL peaks versus initial carrier density, exciting at the lh exciton with $t=12 \mathrm{ps}$. In this case, we observe that the unpolarized emission (open triangles) lies at slightly higher energies than the polarized one (closed triangles), in concordance with the small, but not

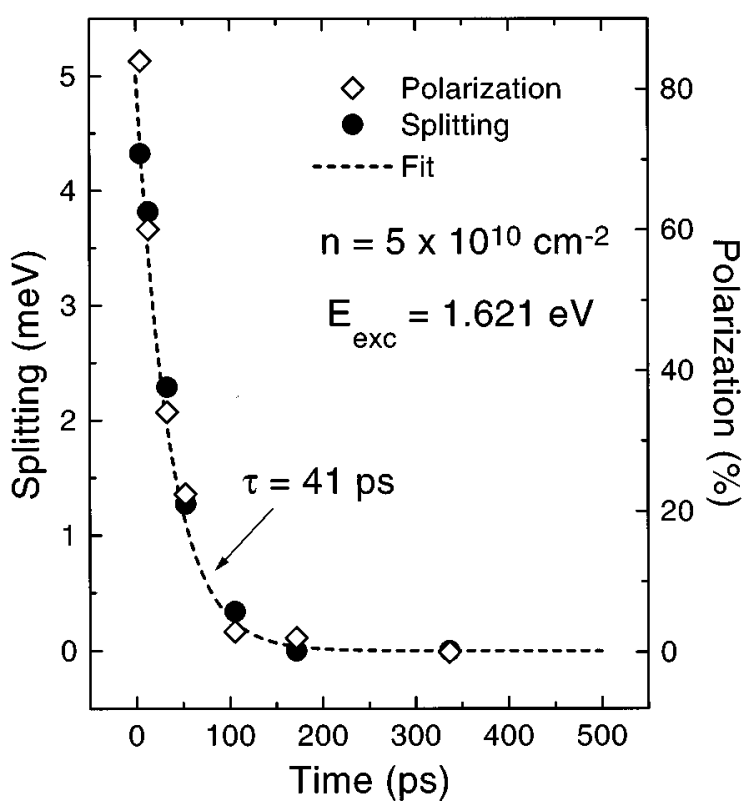

FIG. 4. Time evolution of the PL splitting ( $)$ and polarization $(\diamond)$ for an initial carrier density of $5 \times 10^{10} \mathrm{~cm}^{-2}$ exciting at 1.621 $\mathrm{eV}$. The dashed line depicts the best fit to a monoexponential decay with a time constant of 41 ps.

negligible, value of $P$, which amounts to $-10 \% .^{6}$ The negative value of $P$ corresponds to the fact that exciting at the lh-exciton energy with $\sigma^{+}$polarization, after relaxation of $-1 / 2 \mathrm{lh}$ to $3 / 2 \mathrm{hh}$, the population of $-1 \mathrm{hh}$ excitons is larger than that of +1 hh excitons. The adequacy of the theory to explain the experimental results is also confirmed by the results obtained with Eq. (2) for $P=-10 \%$, which are depicted in the inset as solid $\left(\sigma^{+}\right)$and open $\left(\sigma^{-}\right)$squares for an excitonic density of $9.5 \times 10^{10} \mathrm{~cm}^{-2}$. Although the theory gives again a less pronounced dependence (not shown) on excitation power than the experimental one displayed in the inset, it obtains that both excitons redshift. Furthermore, the calculated shift of the +1 excitons and the splitting amount to $2 \mathrm{meV}$ and $-0.4 \mathrm{meV}$, respectively, in very good agreement with the experimental values.

The theory predicts [neglecting the second term in Eq. (1)] that the splitting between +1 and -1 excitons is proportional to the difference between spin-up $\left(n^{+}\right)$and spindown $\left(n^{-}\right)$populations and therefore proportional to the degree of polarization $\left[P=\left(n^{+}-n^{-}\right) /\left(n^{+}+n^{-}\right)\right]$. This dependence is also borne out from the experiments, as shown in Fig. 4: the time dependence of the splitting (solid points) and of the degree of polarization (open diamonds), for a carrier density of $5 \times 10^{10} \mathrm{~cm}^{-2}$, are strongly correlated and both show a monoexponential decay with a time constant of $40 \mathrm{ps}$. When the $n^{+}$and $n^{-}$populations become comparable, and therefore $P \rightarrow 0$, the splitting vanishes as a consequence of the convergence of the +1 and -1 excitons towards the same energy, as predicted by Eqs. (1) and (2). The reduction of $P$ with time arises from spin-flip processes of the excitons, which are believed to be driven by exchange interaction between the electron and hole composing the exciton. ${ }^{1,8}$ This intraexcitonic exchange interaction does not break the symmetry between spin +1 and spin -1 excitons and has a very 
weak influence in the exciton energy levels, but it is responsible for the time decay of the splitting.

Finally, we would like to mention that the experiments corroborate the theoretical predictions concerning the relative strength of the electron-hole vertex $\left(I_{1}\right)$ and of the electron(hole)-electron(hole) $\left(I_{2}\right)$ self-energy corrections: the splitting grows with increasing initial carrier density corresponding to $I_{1} / I_{2}=6.28 / 4.76>1$ [see Eqs. (A4) and (A12) in Ref. 9]. The possible reversal of this inequality by an external perturbation could have important consequences in the state of polarization of the excitonic gas. The variation of the total energy with the degree of polarization $P$ can be written as

$$
\frac{\partial E_{T}}{\partial P}=\frac{2 e^{2} n^{2}}{\epsilon a}\left(I_{1}-I_{2}\right)(1-f) P .
$$

Taking into account that $f \ll 1$, if $I_{1}>I_{2}$, Eq. (3) predicts that the system prefers to have zero polarization because there it attains an energy minimum. However, if an external perturbation, such as strain, electric field, etc., could cause that $I_{2}>I_{1}$ then the excitonic gas would prefer to be polarized. $^{16}$

In summary, we have shown that the spin splitting observed in a polarized two-dimensional exciton gas originates from the interexcitonic interaction among electrons and holes, forming the excitons. The magnitude of the splitting depends on the process of excitonic formation, it grows with increasing excitonic density and is firmly correlated with the degree of polarization of the system. Its time evolution is determined by the additional intraexcitonic exchange interaction.

\section{ACKNOWLEDGMENTS}

This research was supported in part by the Comisión Interministerial de Ciencia y Tecnología of Spain under Contract No. MAT 94-0982-C02 and by the Commission of European Communities under Contract Ultrafast CHRX-CT930133.
${ }^{1}$ T.C. Damen, L. Viña, J.E. Cunningham, J. Shah, and L.J. Sham, Phys. Rev. Lett. 67, 3432 (1991).

${ }^{2}$ J.B. Stark, W.H. Knox, and D.S. Chemla, Phys. Rev. B 46, 7919 (1992).

${ }^{3}$ B. Dareys, X. Marie, T. Amand, J. Barrau, Y. Shekun, I. Razdobreev, and R. Planel, Superlattices Microstruct. 13, 353 (1993).

${ }^{4}$ T. Amand, X. Marie, B. Baylac, B. Dareys, J. Barrau, M. Brousseau, R. Planel, and D.J. Dunstan, Phys. Lett. A 193, 105 (1994).

${ }^{5}$ A. Vinattieri, J. Shah, T.C. Damen, K.W. Goosen, L.N. Pfeiffer, M.Z. Maialle, and L.J. Sham, Appl. Phys. Lett. 63, 3164 (1993).

${ }^{6}$ L. Muñoz, E. Pérez, L. Viña, and K. Ploog, Phys. Rev. B 51, 4247 (1995).

${ }^{7}$ G. Bastard and R. Ferreira, Surf. Sci. 267, 335 (1992).

${ }^{8}$ M.Z. Maialle, E.A. de Andrada e Silva, and L.J. Sham, Phys. Rev. B 47, 15576 (1993).

${ }^{9}$ J. Fernández-Rossier, C. Tejedor, L. Muñoz, and L. Viña, Phys. Rev. B (to be published).
${ }^{10}$ In this work we denote the third component of the exciton angular moment with the word spin.

${ }^{11}$ L. Muñoz, E. Pérez, L. Viña, J. Fernández-Rossier, C. Tejedor, and K. Ploog, Solid-State Electron. 40, 755 (1996).

${ }^{12}$ N. Peyghambarian, H.M. Gibbs, J.L. Jewell, A. Antonetti, A. Migus, D. Hulin, and A. Myssyrowicz, Phys. Rev. Lett. 53, 2433 (1984).

${ }^{13}$ D. Hulin, A. Myssyrowicz, A. Antonetti, A. Migus, W.T. Masselink, H. Morkoc, H.M. Gibbs, and N. Peyghambarian, Phys. Rev. B 33, 4389 (1986).

${ }^{14}$ S. Schmitt-Rink, D.S. Chemla, and D.A.B. Miller, Phys. Rev. B 32, 6601 (1985).

${ }^{15}$ H. Haug and S. Schmitt-Rink, Prog. Quantum Electron. 9, 3 (1984).

${ }^{16} \mathrm{~J}$. Fernández-Rossier and C. Tejedor, in Proceedings of the 23rd Interntional Conference on the Physics of Semiconductors, edited by M. Scheffler and R. Zimmermann (World Scientific, Singapore, 1996), p. 2463. 